\section{Silicon-based electrically driven microcavity LED}

J. Potfajova, J.M. Sun, S. Winnerl, T. Dekorsy, W. Skorupa, B. Schmidt, M. Helm, S. Mantl and U. Breuer

A silicon $p n$-diode was embedded into a microcavity composed of a buried metal silicide as bottom reflector and a $\mathrm{Si} / \mathrm{SiO}_{2}$ Bragg mirror as top reflector. Spectral narrowing and an increased intensity of the $\mathrm{Si}$ bandgap electroluminescence was observed.

Introduction: The realisation of Si-based, electrically driven light emitters is a key requirement for the implementation of low-cost Si-based optoelectronics [1]. Realising Si-based light sources in a process technology compatible with mainstream microelectronics technology is one of the big challenges of semiconductor technology. Because of its indirect bandgap $\mathrm{Si}$ has a low radiative recombination rate, leading to efficiencies of the bandgap electroluminescence (EL) in the range of $10^{-6}[2,3]$. Recently, different approaches have led to an increase of the power efficiency of the bandgap EL by more than three orders of magnitude up to values of $0.1-1 \%$ [4-6]. These approaches are based on $p n$-diodes, where either the non-radiative lifetime is increased by using high-purity floatzone $\mathrm{Si}$, combined with surface texturing to improve the outcoupling efficiency [5], or where specific defects introduced by ion implantation enhance the radiative recombination rate through carrier confinement effects $[4,6]$. However, the spectral width and temporal response of these devices still constrains their practical application. One possible route for a further enhancement of the efficiency of these devices is a photonic confinement of the emitting layer. III-V semiconductor based LEDs gained significantly in performance by incorporation into microcavities (MCs) [7]. Planar MCs enhance the brightness, efficiency and directionality of the emission from a high-index material and lead to more than an order of magnitude increase in the spectral power density [8]. An MC consists of a cavity with $i \times \lambda_{M C} / 2 n$ thickness ( $i$ integer, $\lambda_{M C}$ resonance wavelength of the MC, $n$ refractive index of the cavity material) embedded between two highly reflecting mirrors. For III-V LEDs molecular epitaxy growth allows the deposition of Bragg mirrors, which act simultaneously as electrical contacts to the active layer. This is a technological problem for Si-based devices, where the Bragg mirrors have to be fabricated from insulating dielectric materials thus inhibiting to electrically drive the active layer. Previously, electrically driven MCs were realised, where the active layer and the Bragg mirror consisted of porous $\mathrm{Si}[9,10]$. These devices suffered from the low stability of porous Si under highvoltage current injection. Here we present a proof-of-principle of an electrically driven MC based on bulk $\mathrm{Si}$ as active layer.

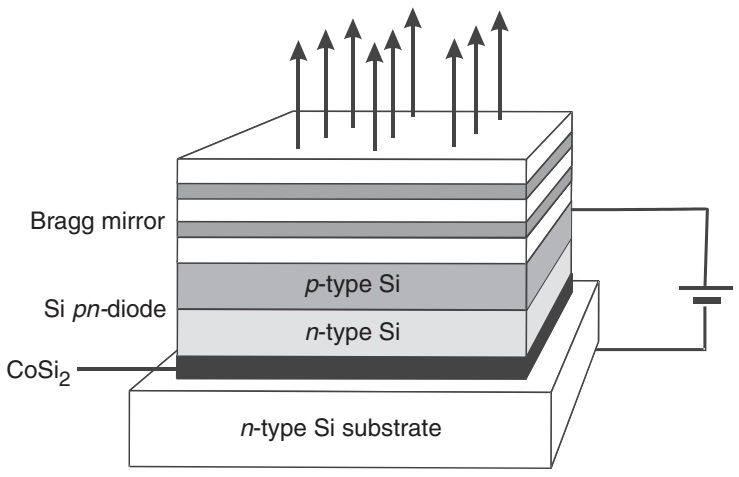

Fig. 1 Schematic diagram of Si-based microcavity

Device preparation: The device structure is shown schematically in Fig. 1. It consists of a buried $\mathrm{CoSi}_{2}$ layer, which acts as the bottom mirror and the bottom electrical contact, the active layer containing the $p n$-diode, and a top Bragg mirror consisting of 2.5 pairs of $\mathrm{Si} / \mathrm{SiO}_{2}$. The $\mathrm{CoSi}_{2}$ layer is formed by ion beam synthesis based on $\mathrm{Co}^{+}$implantation into $n$-doped (100) $\mathrm{Si}$ and subsequent annealing $\left(1100^{\circ} \mathrm{C}\right.$ for $\left.20 \mathrm{~s}\right)$ [11]. The top crystalline $\mathrm{Si}$ layer is overgrown using molecular beam epitaxy to a total thickness of $370 \mathrm{~nm}$, corresponding to $1 \times \lambda_{M C} / n$ at a wavelength of $1115 \mathrm{~nm}$. The $\mathrm{Si}$ layer is uniformly $n$-doped $\left(1.8 \times 10^{17} \mathrm{~cm}^{-3}\right)$ by implanting $\mathrm{P}^{+}$ions and subsequent annealing. The $\mathrm{Si} p n$ diode is formed by $\mathrm{B}^{+}$ion implantation ( $25 \mathrm{keV}$ energy, dose of $4 \times 10^{15} \mathrm{~cm}^{-2}$ ) and hightemperature annealing $\left(1050^{\circ} \mathrm{C}\right.$ for $\left.10 \mathrm{~min}\right)$ into the MBE grown $n$ doped Si top layer. An Al ring contact of $1 \mathrm{~mm}$ inner diameter is lithographically patterned into the evaporated contact layer. The top Bragg mirror is fabricated by sputter deposition of alternating $\mathrm{SiO}_{2}$ layers of $185 \mathrm{~nm}$ thickness and amorphous $\mathrm{Si}$ layers of $75 \mathrm{~nm}$ thickness. Locally the top Bragg mirror is removed above the $\mathrm{Al}$ ring to contact the diode from the top, while the bottom diode contact is provided through the back to the low-resistivity $n$-type substrate and the $\mathrm{CoSi}_{2}$ layer.

Electrical characteristics: Rather than being diode-like the currentvoltage characteristic shows a nearly ohmic behaviour with a resistivity of approximately $3 \Omega$. Secondary ion mass spectroscopy of the diode reveals that this is related to $\mathrm{B}$ diffusion from the projected range of $60 \mathrm{~nm}$ down to the $\mathrm{CoSi}_{2}$ contact, probably due to a high defect density of the MBE grown Si layer. Nevertheless, EL is only observed when a forward bias larger than $1.2 \mathrm{~V}$ is applied to the diode, as expected for current injection into the $p n$ diode. In comparison to diodes with $0.1 \%$ power efficiency prepared by the same technique on high-quality $\mathrm{Si}$, the EL intensity of devices without top Bragg mirror at the same current densities dropped by two orders of magnitude, owing to the almost short-circuited $p n$-diode.

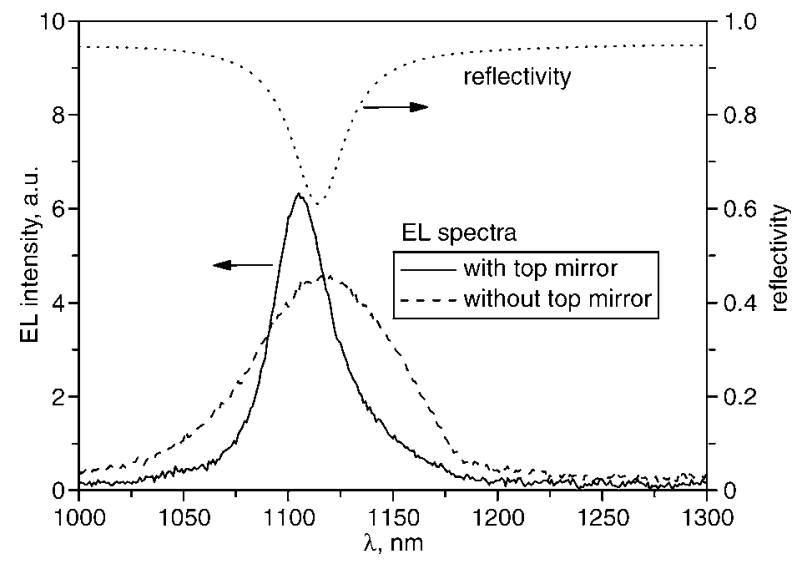

Fig. 2 Electroluminescence spectrum of Si pn-diode

with top Bragg mirror

--- without to Bragg mirror

- . - - reflectivity spectrum of microcavity

EL spectra: Fig. 2 shows the room-temperature EL spectrum of devices without and with Bragg mirror at a current of $500 \mathrm{~mA}$. Additionally the reflectivity spectrum measured within the Al ring contact of a diode is shown, exhibiting the clear signature of a microcavity. The top Bragg mirror has a reflectivity of $94 \%$. At the position matching $\lambda_{M C}=1115 \mathrm{~nm}$ the reflectivity drops to $60 \%$. This spectrum agrees with the modelling of the $\mathrm{MC}$ using a value of $75 \%$ for the reflectivity of the $\mathrm{Si} / \mathrm{CoSi}_{2}$ interface. The bandgap EL spectrum of the device without Bragg mirror shows the same spectrum as previously reported for high-efficiency diodes $[4,6]$. The linewidth of the spectrum is $84 \mathrm{~nm}$. With the top-Bragg mirror the linewidth is decreased to $35 \mathrm{~nm}$ while the peak intensity is significantly increased at $\lambda_{M C}$. The maximum of the EL is shifted by $10 \mathrm{~nm}$ to lower wavelengths as compared to the dip in the reflectivity spectrum. This shift is within the range of scattering of the reflectivity minimum position over the processed wafer owing to non-uniformity of the layer thicknesses in the sputter-deposited Bragg mirror.

Conclusions: We have demonstrated a proof-of-principle of an electrically driven Si microcavity LED. Several possibilities for a further improvement of the output of the MC can be envisioned: because of the $\mathrm{B}$ diffusion the $p n$-junction is spread across the $\mathrm{MC}$ active layer; the optimum coupling of the light emission into the microcavity mode, is achieved, however, when the $p n$-junction is placed in an antinode of the electric field distribution in the MC. This can be achieved by using rapid thermal annealing after the $\mathrm{B}^{+}$implantation, which prevents the strong B diffusion as observed in the present case. 
Further improvement is expected by better matching of the bottom and top reflectivity of the microcavity, e.g. with a $\mathrm{SiO}_{2} / \mathrm{Si}_{3} \mathrm{~N}_{4}$ top mirror, which allows more accurate balancing of the top mirror reflectivity due to the lower refractive index contrast as compared to $\mathrm{Si} / \mathrm{SiO}_{2}$. An improvement of the top $\mathrm{Si}$ quality with a longer nonradiative lifetime could be gained by wafer-bonding methods. A threedimensional confinement could be achieved by lateral patterning of the active layer of the MC into a photonic crystal structure.

(C) IEE 2004

Electronics Letters online no: 20040574

26 March 2004 doi: 10.1049/el:20040574

J. Potfajova, J.M. Sun, S. Winnerl, T. Dekorsy, W. Skorupa, B. Schmidt and M. Helm (Institute of Ion Beam Physics and Materials Research, Forschungszentrum Rossendorf, PO Box 510119, 01314 Dresden, Germany)

E-mail: t.dekorsy@fz-rossendorf.de

S. Mantl (Institute of Thin Films and Interfaces, Forschungszentrum Jülich, 52425 Jülich, Germany)

U. Breuer (Central Division of Analytical Chemistry, Forschungszentrum Jülich, 52425 Jülich, Germany)

\section{References}

1 Canham, L.: 'Gaining light from silicon', Nature, 2000, 408, p. 411
2 Sveinbjörnsson, E.Ö, and Weber, J.: 'Room temperature electroluminescence from dislocation rich silicon', Appl. Phys. Lett., 1996, 69, pp. 2686-2688

3 Kveder, V.V., Steinman, E.A., Shevchenko, S.A., and Grimmeiss, H.G.: 'Dislocation-related electroluminescence at room-temperature in plastically deformed silicon', Phys. Rev. B, 1995, 51, pp. 10520-10526

4 Ng, W.L., Lourenco, M.D., Gwilliam, R.M., Ledain, S., Shao, G., and Homewood, K.P.: 'An efficient room temperature silicon based light emitting diode', Nature, 2001, 410, pp. 192-194

5 Green, M.A., Zhao, J., Wang, A., Reece, P.J., and Gal, M.: 'Efficient silicon light-emitting diodes', Nature, 2001, 412, pp. 805-808

6 Sun, J.M., Dekorsy, T., Skorupa, W., Schmidt, B., and Helm, M.: 'Origin of anomalous temperature dependence and high efficiency of silicon light-emitting diodes', Appl. Phys. Lett., 2003, 83, pp. 3885-3887

7 Benisty, H., De Neve, H., and Weisbuch, C.: 'Impact of planar microcavity effects on light extraction-Part I: basic concepts and analytical trends', IEEE J. Quantum Electron., 1998, 34, pp. 1612-1631

8 Hunt, N.E.J., Schubert, E.F., Logan, R.A., and Zydzik, G.J.: 'Enhanced spectral power density and reduced linewidth at $1.3 \mu \mathrm{m}$ in an InGaAsP quantum well resonant-cavity light-emitting diode', Appl. Phys. Lett., 1992, 61, pp. 2287-2289

9 Araki, M., Koyama, H., and Koshida, N.: 'Controlled electroluminescence spectra of porous silicon diodes with a vertical optical cavity', Appl. Phys. Lett, 1996, 69, pp. 2956-2958

10 Chan, S., and Fauchet, P.M.: 'Tunable, narrow, and directional luminescence from porous silicon light emitting devices', Appl. Phys. Lett., 1999, 75, pp. 274-276

11 Mantl, S.: 'Ion-beam synthesis of epitaxial silicides-fabrication, characterization and applications', Mater. Sci. Rep., 1992, 8, pp. 1-95 